\title{
Blowout Limits of Turbulent Jet Diffusion Flames for Arbitrary Source Conditions
}

\author{
Werner J. A. Dahm* and Avrum G. Mayman $\dagger$ \\ University of Michigan, Ann Arbor, Michigan 48109
}

\begin{abstract}
We present a formulation for the blowout limits of turbulent jet diffusion flames issuing from sources with arbitrary geometries and exit conditions into otherwise quiescent environments. It is argued that, while the liftoff characteristics of turbulent diffusion flames appear likely to be controlled by the straining out of flame sheets, the molecular mixing rate at the flame tip controls their blowout characteristics. The concept of a "far-field equivalent source" is introduced, and the local molecular mixing rate in the flow is expressed in terms of the associated far-field scaling laws. Blowout is expected when a resulting algebraic expression reaches a critical value. Results of a "flip" experiment verify the far-field equivalent source formulation. Measurements of the blowout limits over a range of geometries, fuels, and diluents show good agreement with the predictions from this formulation.
\end{abstract}

\section{Introduction}

$\mathbf{T}$ URBULENT jet diffusion flames form one of the simplest and most commonly used methods for bringing a fuel and oxidizer together in combustion applications. However, the range of operating conditions over which stable combustion can be maintained in such a flame is limited by two self-extinction phenomena generally referred to as "liftoff" and "blowout." In practice, if the fuel flow rate is increased beyond a critical limit, the flame "lifts off" from its source and restabilizes at some distance downstream, with accompanying changes in its soot and radiation characteristics as well as other flame properties. Further increasing the flow rate increases the liftoff height until a second limit is reached at which the flame "blows itself out" entirely. Despite the obvious practical importance of these turbulent combustion stability phenomena, the present understanding of both the liftoff and blowout limits is almost entirely empirical. Even their underlying physical mechanisms have not yet been clearly established. As a consequence, it is not possible to identify a priori the operating limits over which a stable flame can be maintained in a combustion system that departs significantly from conventional design practices. Moreover, since scaling laws for these flame stability limits are as yet unknown for all but the simplest configurations, it is currently not even possible to determine the liftoff and blowout limits of a practical combustion system from measurements on a scaled-down configuration.

The past few years have seen a renewed interest in these aspects of turbulent diffusion flame dynamics, and as a result several fundamentally different underlying physical mechanisms have been proposed to explain the liftoff and blowout phenomena. ${ }^{1-8}$ However, most of this work has been restricted to the single relatively simple configuration formed by a fuel issuing from a round tube. On the one hand, real combustion systems seldom consist of such relatively simple configurations. More importantly, this has ignored potentiaily insightful features of the combustion stability limits of other reacting

Received Sept. 21, 1988; revision received Jan. 13, 1989. Copyright (C) 1988 American Institute of Aeronautics and Astronautics, Inc. All rights reserved.

${ }^{*}$ Assistant Professor, Aerospace Engineering. Member AIAA.

$\nmid$ Currently at Rockwell Space Systems, Downey, CA. shear flows for which the underlying similarity scaling is relatively well-understood. In a notable exception, Kalghatgi ${ }^{3}$ has measured the blowout limits of crossflowing turbulent jets and correlated these by treating the combustion as a premixed flame propagating at an apparent turbulent flame speed through the mean concentration field against the mean velocity field. More recently, Dahm and Dibble ${ }^{9,10}$ examined the liftoff and blowout limits of coflowing turbulent jet diffusion flames, and found that an observed reduction in the blowout limits with increasing coflow velocity could be accurately correlated by an entirely different blowout mechanism proposed by Broadwell et al. ${ }^{8}$

In this paper, we extend the "mixing rate mechanism" from Ref. 8 to jet diffusion flames issuing from steady sources with arbitrary exit conditions into a quiescent environment, as might be typical of practical combustion systems, and demonstrate that the resulting general expression correctly predicts the blowout characteristics of relatively complex sources. Section II begins with an overview of the mixing and combustion processes in turbulent diffusion flames as viewed in a Lagrangian frame, from which two different environments for combustion are identified. It is argued that extinction of one of these controls the flame liftoff characteristics, while the other governs the blowout characteristics. The local molecular mixing rate in the flow is then identified as a potential mechanism by which chemical reactions in the latter can be extinguished, and from this a general expression is formulated for the blowout characteristics of turbulent jet diffusion flames by introducing the notion of a "far-field equivalent source." In Sec. III, experimental results for the blowout limits of jet diffusion flames formed by relatively complex sources are presented which confirm the equivalent source representation and show good quantitative agreement with predictions from this formulation over the entire range of geometries, fuels, and diluents investigated.

\section{Formulation}

A. Lagrangian Description of Jet Mixing and Combustion

Broadwell and Breidenthal ${ }^{11}$ originally proposed a Lagrangian description of the mixing and chemical reaction processes in a turbulent shear layer to explain experimental results obtained in that flow. A similar description for turbulent jets was 
used by Broadwell ${ }^{12}$ and Tyson et al. ${ }^{13}$ to account for an observed nitric-oxide dependence on Reynolds number in jet diffusion flames, and by Broadwell et al. ${ }^{8}$ to postulate a mechanism for blowout of simple turbulent jet diffusion flames. Here, this description is extended to distinguish between the physical mechanisms leading to the liftoff and blowout limits, and to formulate the blowout mechanism for jet flames issuing from arbitrary sources.

In this Lagrangian description, a typical fluid element of cold ambient air is followed as it is entrained into the flow and comes in contact with a typical element containing, as is argued below, a mixture of hot reaction products and excess reactants. As this cold entrained air and the mixture of hot products and reactants proceed down the local turbulent cascade, the fluid elements deform and become intertwined, separated by an interface whose topology grows increasingly complex and is subjected to a spectrum of strain rates $\sigma$. The scale of distortions appearing in the interface is originally of the order of the local flow width $\delta$, and as the cascade proceeds includes increasingly smaller scales until reaching the local Kolmogorov scale $\lambda_{K} \sim \delta \cdot R e^{-3 / 4}$. Here $R e \equiv(u \delta / \nu)$ is the local Reynolds number, with $u$ and $\delta$ the local velocity and length scales characterizing the shear at that stage in the flow, and $\nu$ the kinematic viscosity. The spectrum of strain rates on the interface ranges from $u / \delta$, which corresponds to interface distortion scales of the order of $\delta$, up to the local peak value $u / \delta \cdot R e^{1 / 2}$, which corresponds to distortions of the order of the local Kolmogorov scale. Molecular diffusion of species and heat occur across this strained interface in diffusion layers whose local strain-limited thickness ${ }^{14,15}$ is $\lambda_{D} \sim(D / \sigma)^{1 / 2}$, where $D$ represents the appropriate diffusivity for species or heat. For the highest strain rates, this defines the smallest diffusion layer thickness as the local Batchelor scale $\lambda_{B} \sim \lambda_{K} \cdot S c^{-1 / 2}$, where $S c \equiv(\nu / D)$ is the Schmidt number for species diffusion or the Prandtl number for thermal diffusion. Imbedded within these strained diffusion layers are thin reaction layers ("flame sheets") within which combustion occurs, located at a Howarth transformed distance $\lambda_{D} \cdot \operatorname{erf}^{-1}[(\phi-1) /$ $(\phi+1)]$ from the interface, ${ }^{14}$ where $\phi$ is the local stoichiometric mass ratio across the interface, and having a local thickness $\lambda_{R} \sim \lambda_{D} \cdot D a^{-1 / 3}$, where $D a \equiv(k / \sigma)$ is the local Damköhler number with $k$ the overall reaction rate coefficient. ${ }^{15}$ If the local interfacial strain rate becomes large enough, the rate of enthalpy diffusion from the reaction layer can be sufficiently large to cause local extinction of the layer. ${ }^{6,7,16}$ This will be referred to here as the "flame sheet strain-out mechanism."

As the local turbulent cascade nears its end and the smallest distortions having developed in the interface begin to approach the local Kolmogorov scale, the total interfacial area increases dramatically. ${ }^{11}$ Moreover, the resulting separation between adjacent interfacial diffusion layers also becomes of the order of the Kolmogorov scale and these layers must rapidly begin to overlap. Beyond this point, it is no longer appropriate to speak of diffusion and chemical reactions occurring in distinct layers. Instead, continued molecular diffusion will act to homogenize the fluids. If the two fluids are present in proportions far from stoichiometry, then during this homogenization the fuel concentration in the mixture will quickly exceed the flammability limit and chemical reactions will be thermally quenched, leaving a homogeneous mixture of products and unburned reactants. On the other hand, if the two fluids are sufficiently close to stoichiometry, the homogeneous mixture will remain combustible and the lean reactant (air) will be consumed. The resulting mixture of products and excess reactants then comes into contact with fresh entrained ambient air and this entire mixing and combustion process repeats. With each successive repetition, the homogeneous mixture at that stage in the flow becomes decreasingly fuel rich, and increasingly more combustion occurs during homogenization. The flame ends after the required number of stages to bring the homogeneous mixture to a completely combustible composition.

\section{B. Distinction Between the Liftoff and Blowout Mechanisms}

This Lagrangian view identifies two different environments in which combustion occurs in turbulent diffusion flames, namely (1) within strained diffusion layers during the cascade, and (2) during homogenization upon completion of the cascade. If only relatively few repetitions of this molecular mixing and reaction process have been completed (i.e., at early stages in the flame relative to the flame length), the homogeneous mixture will still be quite fuel rich and as a result relatively little chemical reaction can occur during homogenization. Consequently, early in the flame essentially all of the combustion occurs in strained flame sheets. This suggests that liftoff, which typically occurs early in the flame, should be governed by a mechanism leading to extinction of the strained flame sheets, such as the strain-out mechanism above. However, at later stages in the flame, and in particular near the flame end, the homogeneous mixture becomes decreasingly fuel rich and combustion can occur in strained flame sheets and during homogenization. Even if the strain rates in the flow are made large enough to strain out essentially all of the flame sheets, continued combustion in the homogeneous mixture near the flame end must still be extinguished before the flame can blow out. As noted above, however, the flame sheet strain-out mechanism is physically inappropriate for describing extinction of these homogeneous regions. This suggests that, in order for the flame to blow out, there must be a different mechanism capable of extinguishing the reactions occurring during homogenization.

\section{Mixing Rate Mechanism for Arbitrary Source Conditions}

To identify a mechanism capable of blowing the flame out, the Lagrangian time required for each repetition of this molecular mixing and chemical reaction process was examined in Ref. 8, consisting of the time $t_{K}$ required for the inviscid cascade down to the local Kolmogorov scale and the additional time $t_{D}$ required for subsequent homogenization by molecular diffusion across distances of the order of the Kolmogorov scale. It is argued in Ref. 11 that

$$
t_{K} \sim \frac{\delta}{u}\left[1-R e^{-1 / 2}\right]
$$

and

$$
t_{D}-\frac{\delta}{u} S c R e^{-1 / 2}
$$

where $\delta(x)$ and $u(x)$ are the local length and velocity scales characterizing the shear at that stage in the flow. When the local Reynolds number is large, the combined molecular mixing time $t_{m}$ becomes independent of the Reynolds number and the Schmidt number, and is simply $t_{m}-\delta / u$. Note that this suggests that the downstream distance over which the fluid typically progresses during the time required for each repetition of this mixing process is roughly one local flow width $\delta(x)$.

If the corresponding molecular mixing rate $1 / t_{m}$ between the cold entrained air and the hot product mixture in the strainedout diffusion layers during the cascade and near the Kolmogorov scale during homogenization is sufficiently rapid, there will be insufficient time for initiation of the reactions during homogenization before the temperature drops below a critical value for ignition. A characteristic chemical time $t_{c}$ for the onset of reactions can be inferred from the laminar flame speed $S$ and the thermal diffusivity $K$ as $t_{c} \sim K / S^{2}$. Reactions during homogenization would then be extinguished if the local mixing time $t_{m}$ becomes sufficiently fast relative to the ignition time $t_{c}$, namely, when their ratio

$$
\epsilon \equiv \frac{\delta / u}{K / S^{2}}
$$

falls below a critical value. 
Application of Eq. (1) to the jet diffusion flame formed by any arbitrary source requires the local mixing time $\delta / u$ at any downstream location $x$ to be expressed in terms of its source characteristics. Although the $u(x) \sim x^{-1}$ decay of the local centerline velocity and the $\delta(x) \sim x$ linear growth of the local flow width in the self-similar far field of axisymmetric turbulent jets issuing into quiescent environments are well-known, their proper representation for an arbitrary jet source does not appear to be widely known. These can be easily derived by recognizing that details of the jet source such as its geometry or exit profiles cannot remain dynamically relevant at downstream distances that are large in comparison with the scale of the source. Rather, at most only integral quantities derived from these details can be relevant far downstream. Among such integrals, the source mass flux cannot remain relevant since the total mass flux of the jet increases with downstream distance and eventually must dominate any influence of the source mass flux. The total momentum flux $J(x)$ of the jet must, however, remain invariant and equal to the source momentum flux $J_{o}$. Moreover, since far downstream the fluid moving in the jet consists almost entirely of ambient fluid, physical properties of the source fluid also cannot remain important. Following this reasoning, the "far field" of a turbulent jet is defined as distances sufficiently far from the source so that the only dynamically relevant characteristic of the jet source is its momentum flux $J_{o}$. Dimensional reasoning then requires that the characteristic local flow width $\delta(x)$ and velocity $u(x)$ in the far field, which set the local molecular mixing rate $u / \delta$, must scale as

$$
\begin{gathered}
\delta(x) \sim x \\
u(x) \sim\left(J_{o} / \rho_{\infty}\right)^{1 / 2} x^{-1}
\end{gathered}
$$

The axial location $x$ at which extinction of the reactions during homogenization will lead to blowout remains to be specified. We note here that the linear self-similar scaling of axisymmetric turbulent jets simply requires that this must occur in the same position relative to the flame length for any such flame, giving

$$
x \sim L
$$

where $L$ is the flame length. The flame length scaling can be inferred from the scaling for the mixture fraction, which can be shown ${ }^{17}$ from the invariance of the source fluid mass flux integral and self-similarity in the far field to scale as $c(x)$ $\left(x / d^{*}\right)^{-1}$, where

$$
d^{*} \equiv \frac{2 m_{o}}{\left(\pi \rho_{\infty} J_{o}\right)^{1 / 2}}
$$

Here $d^{*}$ is the diameter of a conceptual "far-field equivalent source," through which a homogeneous mixture of the source fluids would flow in the same proportions as the actual source and with the same mass flux $m_{o}$ and momentum flux $J_{o}$ as the actual source. As a consequence, this equivalent source would produce the same $\delta(x), u(x)$, and $c(x)$ in the far field, and would thus have the same flame length and the same mixing rates, and therefore the same blowout characteristics, as the actual source.

The flame length $L$ in Eq. (3) scales with the axial distance at which the mixture fraction reaches stoichiometry, namely, $c(L) \sim 1 /\left(1+\varphi^{\prime}\right)$, where $\varphi^{\prime}$ is the stoichiometric ambient-tosource fluid mass ratio for the homogeneous source fluid mixture, given by

Oxidizer diluent:

$$
\varphi^{\prime}=(1-\mu) \varphi-\mu
$$

Inert diluent:

$$
\varphi^{\prime}=(1-\mu) \varphi
$$

Fuel diluent:

$$
\varphi^{\prime}=(1-\mu) \varphi_{1}+\mu \varphi_{2}
$$

with $\mu$ the mass fraction of diluent in the homogeneous mixture and $\varphi$ the stoichiometric mass ratio for the pure fuel. This gives the flame length scaling in Eq. (3) as

$$
L \sim\left(1+\varphi^{\prime}\right) d^{*}
$$

References 12 and 17 verify that a scaling essentially identical to Eq. (6) correctly correlates the lengths of a wide range of turbulent jet diffusion flames.

Equations (1-6) give the blowout parameter $\epsilon$ as

$$
\epsilon=\frac{\sqrt{\pi}}{2} \frac{\left(d^{*}\right)^{2}(S)^{2}\left(1+\varphi^{\prime}\right)^{2}\left(\rho_{\infty}\right)^{1 / 2}}{\left(J_{0}\right)^{1 / 2}(K)}
$$

where the blowout limits of turbulent jet diffusion flames lifted in the far field should be correlated by a single value of $\epsilon$. It is noteworthy that there are no free parameters involved. The factor $\sqrt{\pi} / 2$ has been introduced so that, for a pure fuel issuing from a round tube with uniform density and velocity profiles, this formulation reduces to the expression in Ref. 8, where blowout was correlated for such simple sources by $\epsilon \approx 4.8$. Here, the blowout characteristics of the flame generated by an arbitrary source have been expressed in terms of the equivalent far-field representation of the source. Note that the factor $\left(1+\varphi^{\prime}\right)^{2}$ in Eq. (7), which results directly from the arguments leading to Eq. (6), differs from that in the corresponding expression in Ref. 8 . We demonstrate in Sec. IV that the factor in the present formulation corrects the errors apparent at large diluent concentrations, for which $\varphi^{\prime}$ is relatively small, in Ref. 8.

\section{Experiments}

\section{A. Experimental Facility}

To test the extent to which the simple expression in Eq. (7) correctly predicts the blowout characteristics of turbulent jet diffusion flames, a set of experiments was conducted to measure the blowout limits of several comparatively complex sources. A family of coaxial jet source geometries was chosen since these allow a fairly wide range of source conditions to be generated. Various combinations of inner and outer nozzle diameters were used to establish differing configurations. Either of two inner nozzles, one with a $3 \mathrm{~mm}$ diam $(3.13 \mathrm{~mm}$ i.d., $3.69 \mathrm{~mm}$ o.d.) and the other with a $5 \mathrm{~mm}$ diam $(4.91 \mathrm{~mm}$ i.d., $5.25 \mathrm{~mm}$ o.d.) were used, together with either of two outer nozzles having diameters of $7 \mathrm{~mm}(7.14 \mathrm{~mm}$ i.d.) and $10 \mathrm{~mm}$ (10.08 $\mathrm{mm}$ i.d.). The individual fuel and diluent gases could each be arranged as either the inner or outer streams. Technical grade methane or ethylene were used for the fuel streams, and carbon dioxide or air as the diluent streams. The gases issued from high flow rate regulators through fine metering valves held at sonic conditions with a constant upstream pressure. A pair of laminar flow meters measured the individual volumetric flow rate for each stream. To permit the contribution of each stream to the total momentum flux $J_{o}$ to be accurately inferred from its measured volumetric flow rate, the nozzle contractions were specifically designed to give essentially uniform velocity profiles over each of the inner and annular exit areas. To achieve this, area ratios for the nozzle contractions ranged from several hundred-to-one to almost a thousand-to-one, for which Thwaites' method estimates indicated that the resulting boundary-layer momentum thicknesses were typically less than $2 \%$ of the exit diameter. A ventilating hood insured that combustion products did not influence the ambient air composition. 


\section{B. "Flip" Experiment}

As a test of the far-field equivalent source representation for formulating the blowout characteristics of arbitrary sources, the blowout stability limits were measured for the coaxial source formed by the $5 \mathrm{~mm}$ and $7 \mathrm{~mm}$ diam nozzles. This geometry was specifically chosen since it produces virtually
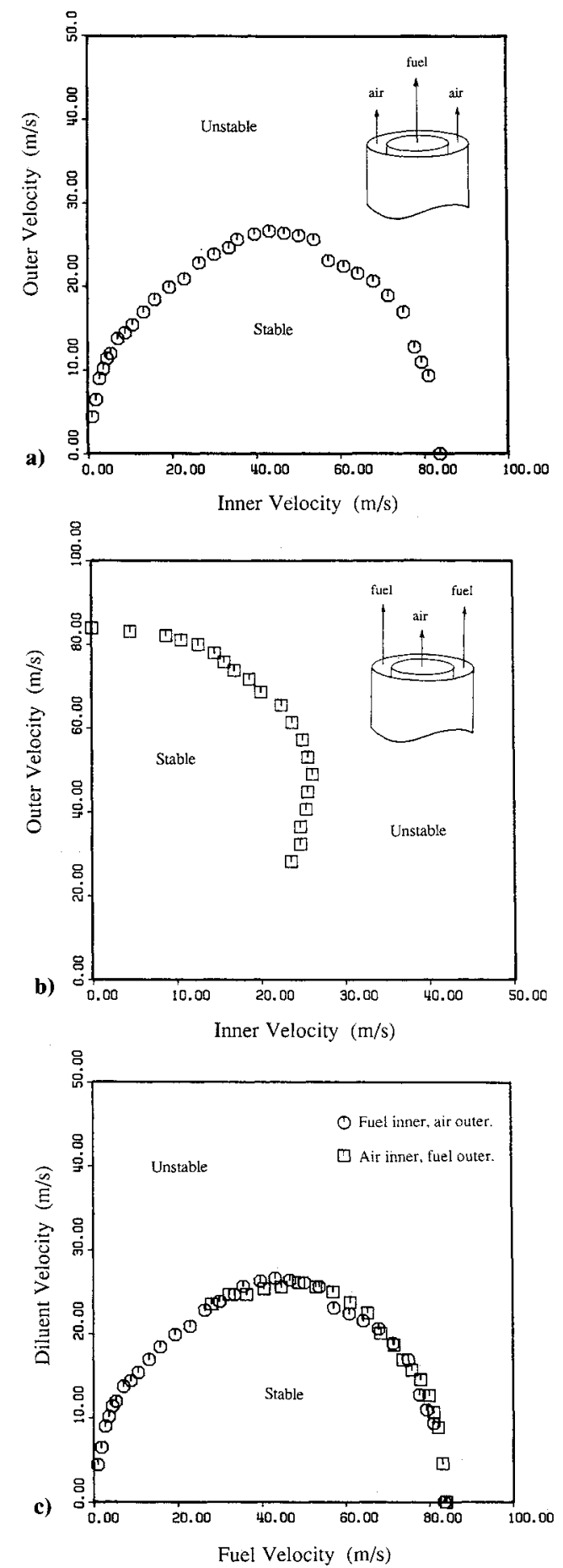

Fig. 1 "Flip" experiment demonstrating validity of the far-field equivalent source concept for turbulent diffusion flame blowout, showing the measured blowout limits of two configurations for which reversing the fuel and diluent streams produces the same equivalent source representation in the far field: a) measured blowout limits for coaxial configuration with inner methane stream $(3 \mathrm{~mm}$ diam) and annular air stream (7 mm diam); b) measured blowout limits for coaxial configuration with inner air stream ( $3 \mathrm{~mm}$ diam) and annular methane stream ( $7 \mathrm{~mm}$ diam); and c) blowout limits from parts a and b compared in terms of the relevant quantities, namely, the fuel and diluent velocities at blowout, verifying that the blowout limits are identical as suggested by Eq. (7). equal exit areas for the inner and annular streams. Consequently, reversing the location of the fuel and diluent between the inner and annular streams would produce very large changes in the density and velocity profiles of the source, but would leave unaltered the corresponding far-field quantities, namely, the source mass flux $m_{o}$, momentum flux $J_{o}$, and diluent mass fraction $\mu$. As a result, Eq. (7) suggests that the fuel and diluent flow rates at blowout should be the same for both of these configurations.

Figures la and $1 \mathrm{~b}$ show the measured blowout limits for each of these two configurations. Note that, since the equivalent representation of the source remains identical for both configurations, the notions of an "inner" stream and an "outer" stream are not relevant in the far field. Following this reasoning, we compare these results in terms of the relevant quantities, namely, the fuel and diluent velocities, in Fig. Ic. In this form, it can be seen that the blowout limits are indeed essentially identical for both configurations. This equivalence provides strong support for the notion that the blowout limits of any complex source are determined entirely by its far-field equivalent representation in Eq. (4).

\section{Comparisons with Eq. (7)}

A contour map of the blowout parameter $\epsilon$ in Eq. (7) computed for the far-field equivalent source representation of the configurations shown in Fig. 1 is presented in Fig. 2. In this and all subsequent calculations, the laminar flame speeds, stoichiometric ratios for the pure fuels, and thermal diffusivities were taken as in Ref. 8. Also shown are the measured blowout limits from Fig. 1c for each of these two configurations. The comparison shows that good quantitative prediction of the measured blowout limits is given by the $\epsilon \approx 4.8$ contour.

The measured blowout limits for a wide range of geometries, fuels, and diluents are presented in Fig. 3 together with the $\epsilon \approx 4.8$ contour for each case from Eq. (7). In Figs. $3 \mathrm{a}$ and $3 \mathrm{~b}$, results are shown for methane and air issuing from different coaxial geometries, while Fig. $3 \mathrm{c}$ shows two cases in which $\mathrm{CO}_{2}$ and methane issue from such coaxial sources. Note that, while the predictions for dilution with air are fairly good, the results for $\mathrm{CO}_{2}$ show larger errors. It is likely that at least some of this error is attributable to the approximate method ${ }^{2,8}$ used to determine the effect of $\mathrm{CO}_{2}$ concentration on the laminar flame speed. In contrast, dilution with air does not affect the laminar flame speed and introduces no such error. Similar results for ethylene and air issuing from a coaxial source are shown in Fig. 3d. In this case, icing of the fuel regulator prevented measurements at high fuel flow rates. Nevertheless, over the range attainable the agreement between the measured blowout limits and the formulation in Eq. (7) is good.

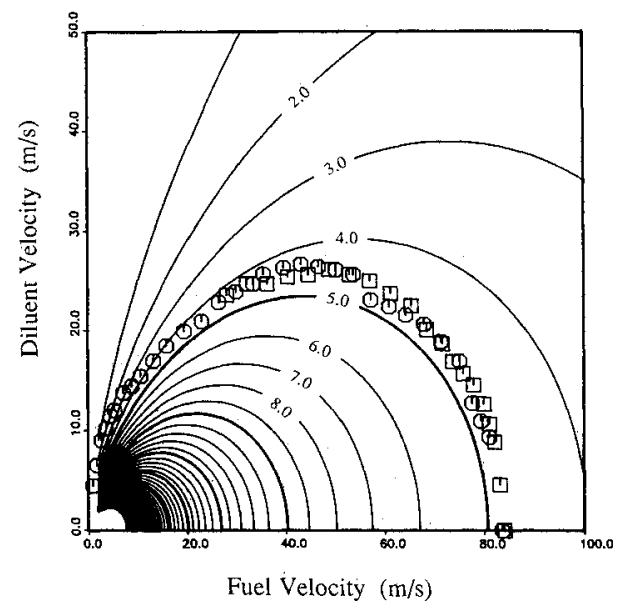

Fig. 2 Contours of the blowout stability parameter $\epsilon$ in Eq. (7) for the configurations shown in Fig. 1, showing comparisons with the measured blowout limits for both configurations. The contour interval is $\Delta \epsilon=1.0$. Blowout is expected at $\epsilon \approx 4.8$. 
a)

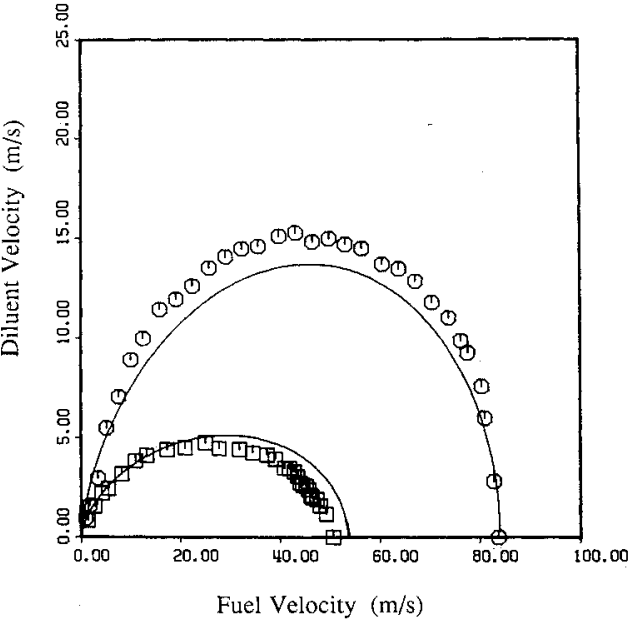

b)

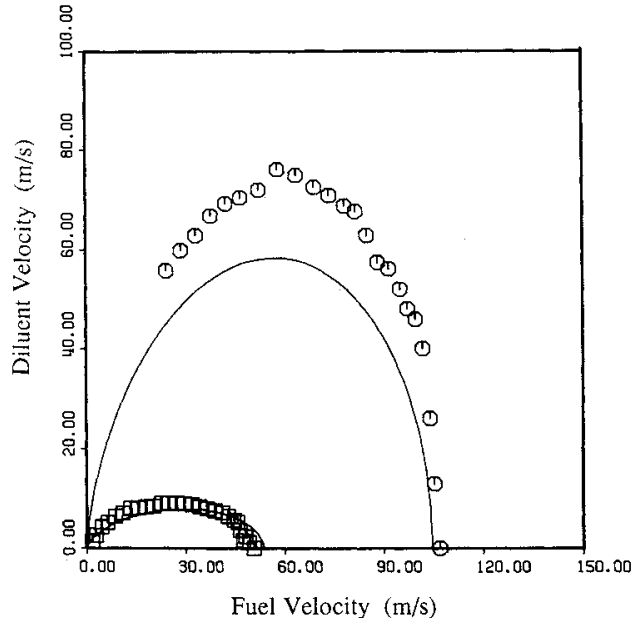

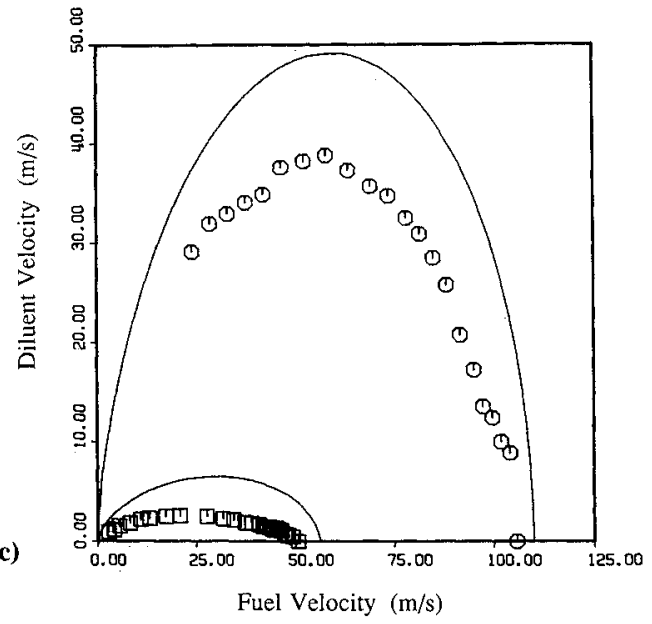

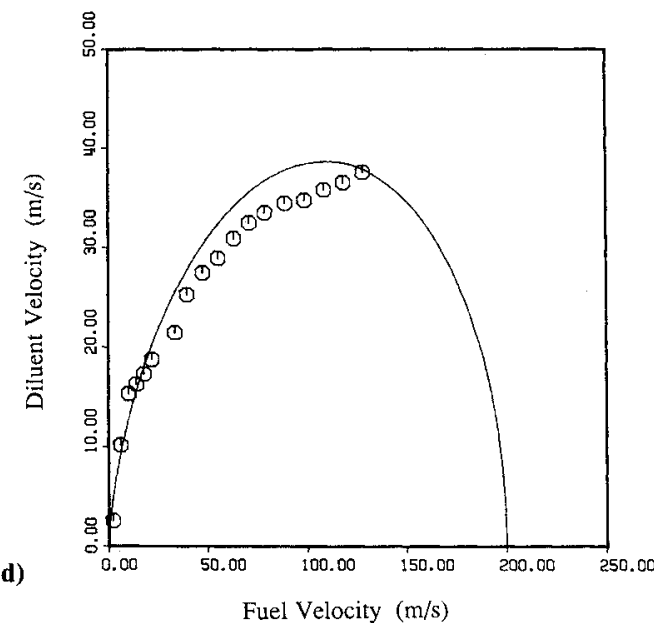

Fig. 3 Comparison of the measured blowout stability limits with the theoretical contour $\epsilon \approx 4.8$ from Eq. (7) for a range of coaxial geometries, fuels, and diluents: a) methane with air diluent. O methane inner, $5 \mathrm{~mm}$; air outer, $10 \mathrm{~mm}$; - Eq. (7). $\square$ methane inner, $3 \mathrm{~mm}$; air outer, $10 \mathrm{~mm}$; - Eq. (7); b) methane with air diluent. $\mathrm{c}$ methane inner, $3 \mathrm{~mm}$; air outer, $7 \mathrm{~mm}$; - Eq. (7). 0 air inner, 3 mm; methane outer, $7 \mathrm{~mm}$; - Eq. (7); c) methane with $\mathrm{CO}_{2}$ diluent. $\square$ methane inner, $3 \mathrm{~mm}$; $\mathrm{CO}_{2}$ outer, $7 \mathrm{~mm}$; - Eq. (7). O $\mathrm{CO}_{2}$ inner, $3 \mathrm{~mm}$; methane outer, $7 \mathrm{~mm}$; - Eq. (7); and d) ethylene with air diluent. $\circ$ ethylene inner, $3 \mathrm{~mm}$; air outer, $7 \mathrm{~mm}$; - Eq. (7).

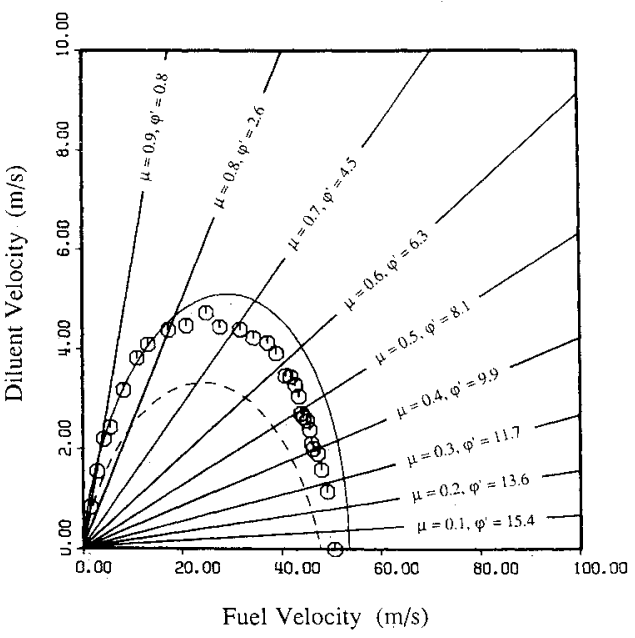

Fig, 4 Comparison of measured blowout limits for a typical case with the $\epsilon \approx 4.8$ contour in Eq. $(7)$ for $\left(\varphi^{\prime}\right)^{2}$ and $\left(1+\varphi^{\prime}\right)^{2}$ factors, showing lines of constant diluent mass fraction $\mu$ and corresponding stoichiometric mixture ratios $\varphi^{\prime}$. O methane inner, $3 \mathrm{~mm}$; air outer, $10 \mathrm{~mm} ;---\left(\varphi^{\prime}\right)^{2} ;$ and $-\left(1+\varphi^{\prime}\right)^{2}$.

\section{Discussion and Conclusions}

The results in Fig. 1 verify that the blowout limits of turbulent jet diffusion flames formed by sources with relatively complex geometries and exit conditions can be accurately de- scribed by the equivalent source representation in Eq. (4). Furthermore, the results in Figs. 2 and 3 suggest that the simple formulation in Eq. (7) gives fairly accurate predictions for the blowout limits of such flames, and support the notion that the molecular mixing rate is the mechanism controlling the blowout characteristics of turbulent diffusion flames.

This view of the blowout mechanism differs fundamentally from that proposed by Kalghatgi, ${ }^{2}$ where an expression that involves the kinematic viscosity of the source fluid is used to correlate the blowout limits of simple jet diffusion flames. In contrast, here we have argued that the physical properties of the source fluid, including its viscosity, should not be dynamically relevant in the far field and therefore should not be involved in determining the blowout limits. [Note that Eq. (7) involves only physical properties of the ambient fluid.] Indeed, the measurements in Fig. 1 appear to support this proposal, and suggest that blowout is controlled entirely by the far-field equivalent representation of the source.

For the simple case of a fuel issuing from a round tube, Eq. (7) reduces to the corresponding expression in Ref. 8, with the notable exception that the factor $\left(1+\varphi^{\prime}\right)^{2}$ appears here in place of $\left(\varphi^{\prime}\right)^{2}$. The difference is small for the typically large $\varphi^{\prime}$ associated with pure hydrocarbon fuels, but when the mass fraction of an oxidizing or inert diluent becomes large, Eqs. (5) show that $\varphi^{\prime}$ can become quite small, with the result being that very significant differences arise in the blowout predictions. To demonstrate this, Fig. 4 compares both expressions with the blowout limits measured for a typical case. Bearing in mind that the diluent mass fraction is constant along straight lines 
emanating from the origin in this figure, the difference between the two formulations can be seen to become quite large as the diluent mass fraction increases. The present formulation more nearly correlates the measured blowout limits, especially at large diluent mass fractions, in this and all other cases investigated. Much of the error apparent at large diluent mass fractions in Fig. 5 of Ref. 8 is due to this difference in the blowout formulations.

These blowout results may also give some insight into the mechanism controlling the liftoff characteristics of turbulent diffusion flames. In Sec. II, it was noted that the axial distance required for completion of each successive repetition of the mixing and reaction process is typically equal to the local flow width $\delta(x)$. Since this picture suggests that relatively little reaction takes place during homogenization until the final stage before the flame tip, it should typically be possible to lift a flame to within about one $\delta(L)$ upstream of the flame tip. The flame should then blow out when the mixing rate at that stage in the flow becomes large enough, in the context of Eq. (7), to extinguish reactions during homogenization. This agrees with observations from simple turbulent jets issuing into a quiescent environment, ${ }^{17,18}$ for which $\delta \approx 0.44 x$ and experiments show that all such flames blow out after lifting to a fixed fraction (roughly half) of their flame height. Moreover, for coflowing turbulent jet diffusion flames, ${ }^{9,10} \delta(L)$ is typically a much smaller fraction of the flame length and indeed experimental observations ${ }^{9}$ show that such flames can be lifted to a significantly larger fraction of their flame length. These simple observations reinforce our present view that, while liftoff may be controlled by the straining out of flame sheets, the molecular mixing rate appears to be the mechanism governing blowout of turbulent diffusion flames.

\section{Acknowledgments}

The work reported here was supported by the Basic Sciences (Combustion) Department of the Gas Research Institute (GRI) under Contract 5087-260-1443. The assistance of R. Tangri in preparation of the figures is appreciated.

\section{References}

'Vanquickenborne, L. and van Tiggelen, A., "The Stabilization Mechanism of Lifted Diffusion Flames," Combustion and Flame, Vol. 10, March 1966, pp. 59-69.

${ }^{2}$ Kalghatgi, G. T., "Blowout Stability of Gaseous Jet Diffusion Flames. Pt. I. In Still Air," Combustion Science and Technology, Vol.
26, No. 6, 1981, pp. 233-239.

${ }^{3}$ Kalghatgi, G. T., "Blowout Stability of Gaseous Jet Diffusion Flames. Pt. II. Effect of Crossflow,' Combustion Science and Technology, Vol. 26, No. 6, 1981, pp. 241-244.

${ }^{4}$ Kalghatgi, G. T., "Liftoff Heights and Visible Lengths of Vertical Turbulent Jet Diffusion Flames in Still Air," Combustion Science and Technology, Vol. 41, 1984, pp. 17-29.

${ }^{5}$ Byggstøyl, S., and Magnussen, B. F., Turbulent Shear Flows 4, Vol. 4, edited by L. S. J. Bradbury et al., Springer, New York, 1985, p. 381 .

6Peters, N., "Local Quenching Due to Flame Stretch and Nonpremixed Turbulent Combustion," Combustion Science and Technology, Vol. 30, No. 1-6, 1983, pp. 1-17.

${ }^{7}$ Peters, N. and Williams, F. A. "Liftoff Characteristics of Turbulent Jet Diffusion Flames," AIAA Journal, Vol. 21, No. 3, 1983, pp. 423-429.

of Turbulent Diffusion Flames," Proceedings of the Twentieth Symposium (International) on Combustion, The Combustion Institute, Pittsburgh, 1984, pp. 303-310.

${ }^{9}$ Dahm, W. J. A., and Dibbler, R. W., "Combustion Stability Limits of Coflowing Turbulent Jet Diffusion Flames," AIAA Paper 88-0538, 1988.

${ }^{10}$ Dahm, W. J. A. and Dibble, R. W., "Coflowing Turbulent Jet Diffusion Flame Blowout," Proceedings of the Twenty-Second Symposium (International) on Combustion, The Combustion Institute, Pittsburgh, 1988, pp. 801-808.

${ }^{11 B r o a d w e l l, ~ J . ~ E . ~ a n d ~ B r e i d e n t h a l, ~ R . ~ E ., ~ " A ~ S i m p l e ~ M o d e l ~ o f ~}$ Mixing and Chemical Reaction in a Turbulent Shear Layer,' Journal of Fluid Mechanics, Vol. 125, 1982, pp. 397-410.

${ }^{12}$ Broadwell, J. E., "A Model of Turbulent Diffusion Flames and Nitric-Oxide Generation. Pt. I," TRW Document 38515-6001-UT-00, 1982.

${ }^{13}$ Tyson, T. J., Kau, C. J., and Broadwell, J. E., "A Model of Turbulent Diffusion Flames and Nitric-Oxide Generation. Pt. II," TRW Document 38515-6001-UT-00, 1982.

${ }^{14}$ Mable, F. E., and Broadwell, J. E., "The Coherent Flame Model for Turbulent Chemical Reactions,"' Project SQUID Technical Rept. TRW-9-PU, 1977.

${ }^{15}$ Carrier, G. F., Fendell, F. E., and Marble, F. E., "The Effect of Strain Rate on Diffusion Flames," SIAM Journal of Applied Mathematics, Vol. 28(2), 1975, pp. 463-500.

${ }^{16}$ Liñán, A., "The Asymptotic Structure of Counterflow Diffusion Flames for Large Activation Energies,' Acta Astronautica, Vol. 1, No. 7/8, 1974, pp. 1007-1039.

${ }^{17}$ Dahm, W. J. A., "Entrainment, Mixing, and Chemical Reaction in the Far Field of Turbulent Jets," Ph.D. Thesis, California Institute of Technology, Pasadena, CA, 1985.

${ }^{18}$ Dahm, W. J. A., and Dimotakis, P. E., "Measurements of Entrainment and Mixing in Turbulent Jets," AIAA Journal, Vol. 25, No. 9, 1987, pp. 1216-1223. 